Review

\title{
Chronic wounds and their management and application in a contemporary Sri Lankan hospital setting
}

\author{
JMRWW Wadinamby \\ Sri Lankan Journal of Infectious Diseases 2013 Vol.3(2):2-16 \\ DOI: http://dx.doi.org/10.4038/sljid.v3i2.5974
}

Key words: chronic wounds; pathogenesis; repair; microbiology

\begin{abstract}
The pathogenesis of chronic wounds is complex, resulting in major difficulties in the management of such wounds. Diabetes mellitus, venous ulceration and pressure sores contribute to the rapidly increasing burden of chronic wounds, not only to the patient but also to the health care system. Improved understanding of the factors which contribute to non healing of wounds and application of appropriate management modalities, including a multidisciplinary team approach, would help reduce the time for healing of such wounds. Colonization and infection of chronic wounds is only one component of the pathogenesis which however requires access to a modern microbiology laboratory. The setting up of chronic wound clinics in 3 hospitals in Sri Lanka may provide a model for improving the outcome of such wounds in a relatively under resourced setting.
\end{abstract}

\section{Introduction}

Microbiologically, the primary function of normal intact skin is to prevent invasion by potential pathogens that live on the skin and pathogens from other exogenous sources from entering the body. Loss of skin integrity, namely 'the wound' provides a suitable environment for microbial colonization and propagation that could harm the body. However, the abundance and diversity of microorganisms in any wound will be influenced by factors such as wound type, depth, location and quality, level of tissue perfusion and the antimicrobial efficacy of the host immune response. The microflora associated with clean surgical wounds would be expected to be minimal and limited to skin commensals. In comparison, the presence of foreign material and devitalized tissue in a traumatic wound facilitates microbial proliferation including regional flora as well as flora from other sources. Early antibiotic therapy and surgical debridement would minimize such complications. ${ }^{1}$

\section{Wound healing mechanisms}

\footnotetext{
${ }^{1}$ Base Hospital, Angoda (Infectious Diseases Hospital), Gothatuwa New Town, Angoda, Sri Lanka
}

Address for correspondence: DrRohini Wadinambe, Base Hospital, Angoda (Infectious Diseases Hospital), Gothatuwa New Town, Angoda, Sri Lanka. Email: rohiniwadanamby@gmail.com Tel: +94 (0)777258366 
Our understanding of wound-healing mechanisms has tremendously improved over the past decade with the identification of the role of different cell types and description of different chemokines involved in wound healing.

Traditionally, wound healing is divided into three phases: the inflammatory phase, the proliferation phase, and the remodelling phase. In the inflammatory phase, it involves an interaction among blood vessels (platelets, macrophages, neutrophils, endothelial cells, and smooth muscle cells), epidermis (keratinocytes, melanocytes, and Langerhan cells), adnexal structures (outer root sheath cells and hair dermal papilla cells), dermis (fibroblasts and myofibroblasts), nervous system (neurons) and subcutaneous fatty layers (adipocytes). ${ }^{2}$ Thrombus formation - which requires interaction between endothelial cells, platelets, and coagulation factors - achieves haemostasis after tissue injury, in the latter part of the inflammatory phase. Trapped cells within the clot, predominantly platelets, trigger an inflammatory response by the release of vasodilators and chemo attractants and activation of the complement cascade. ${ }^{3}$ During the coagulation phase after injury, platelets initiate the wound healing process by releasing a number of soluble mediators, including platelet-derived growth factor(PDGF), insulin-like growth factor-1 (IGF-1), epidermal growth factor (EGF), fibroblast growth factor (FGF), and transforming growth factor-beta (TGF- $\beta$ ). These rapidly diffuse from the wound and inflammatory cells are drawn to the area of the injury. ${ }^{4}$ This whole process takes place during the inflammatory phase.

The inflammatory phase is initiated by platelet degranulation and clotting of blood resulting from activation of clotting factors. During this phase, a significant vasodilatation with increased capillary permeability, complement activation, and migration of polymorphonuclear leukocytes (PMN) and macrophages to the wound site have been observed. The neutrophils and macrophages destroy bacteria by engulfing them and release proteases such as elastase and collagenase, which degrade damaged extracellular matrix (ECM) components. They also secrete additional growth factors TGF- $\beta$, TGF- $\alpha$, heparin-binding epidermal growth factor (HB-EGF), and basic fibroblast growth factor (bFGF). Inflammation is largely regulated by a class of molecules called cytokines which have powerful stimulatory or inhibitory actions on inflammatory cells. ${ }^{4,5}$ Cytokines which were known to influence chemotaxis, proliferation, and differentiation of inflammatory cells into other forms of cells involved in wound healing are now recognized to have a significant role in the wound healing process. Interleukin 1(IL1) and $\mathrm{TNF} \alpha$ stimulate production of proteases by fibroblasts. In addition, $\mathrm{TNF} \alpha$ induces apoptosis in fibroblasts. Macrophages recruit further macrophages and continue to stimulate migration of fibroblasts, epithelial cells, and vascular endothelial cells into the wound to form granulation tissue, which starts on the fifth day following injury. ${ }^{4}$

Chemokines, a group of regulatory proteins, are also known to influence the healing process. There are about 40 chemokines. Primarily, these elements regulate trafficking of leukocyte populations during normal health. The development described in the four major cell groups, recruitment and activation of neutrophils, lymphocytes, macrophages, eosinophils, and basophils during inflammation are also known to be carried out by chemokines. ${ }^{4}$

In summary, growth factors, cytokines, and chemokines are key molecular regulators of wound healing. They are all proteins or polypeptides, are typically synthesized and released locally, and primarily influence target cells by paracrine actions. ${ }^{4}$

Proliferation and repair of the matrix: Towards the end of the inflammatory process, due to reduction of inflammatory cells in the wound, the fibroblasts, endothelial cells, and 
keratinocytes take over the synthesis of growth factors. These continue to promote cell migration, proliferation, new capillary formation and synthesis of ECM components. Fibroblasts are the key cells involved in the production of ECM. In addition to producing collagen, they produce tenascin, fibronectin and proteoglycans such as hyaluronic acid. Production of ECM is seen clinically as formation of granulation tissue. The combination of new tissue and contraction of surrounding tissues is essential for the healing of ulcers. ${ }^{6}$ While new matrix is synthesised, existing matrix in and around the wound margin is degraded by several enzyme systems such as matrix metalloproteinases (MMP) and plasminogen activators. Research interest has centred on the role of the metalloproteinases, a family of over 14 enzymes with proteolytic activity which include collagenases, gelatinases, and stromelysins. ${ }^{4}$ The effect of these enzymes is regulated by tissue inhibitors, which are believed to be important in healing by preventing excessive matrix degradation. ${ }^{7}$ While some keratinocytes at the wound edge proliferate, others undergo a marked transformation to enable them to phagocytose debris and migrate across the wound bed. Keratinocyte migration coupled with wound contraction results in re-epithelialisation and wound closure. ${ }^{4}$

Remodelling: Once closure of the wound has been achieved, remodelling of the resulting scar takes places over months or years, with a reduction of both cell content and blood flow in the scar tissue. The increased density of fibroblasts and capillaries present in the early phase of healing reduces, primarily through apoptosis. In the final remodelling phase, tensile strength reaches a maximum as cross linking of collagen fibrils plateaus. ${ }^{4}$

The description of wound healing given above reflects the complexities of the biological process of wound healing. When this typical response of healing is impaired or modified, a wound become chronic.

\section{Factors affecting wound healing}

There are many factors which lead to a wound becoming a chronic wound which could operate either locally or in a generalized manner. Local factors include the presence of foreign bodies, tissue maceration, excessive secretions, ischaemia, venous and lymph stasis, dermatitis and infection. Systemic factors are diverse and range from advanced age to malnutrition, diabetes, blood disorders, impaired immunity and renal disease. ${ }^{8}$ It is important to address these factors individually and collectively to achieve wound healing. Wound care practitioners should attempt to remove or reduce the impact of these factors, even though it may not always be possible to do so.

In addition to local and systemic factors that impair healing, reduction in tissue growth factors, an imbalance between proteolytic enzymes and their inhibitors and the presence of senescent cells seem to be particularly important in chronic wounds. ${ }^{8}$ Reduced levels of active growth factors in the wound may partly explain why certain wounds fail to heal as anticipated. Chronic ulcers are known to have reduced levels of PDGF, bFGF, EGF and TGF- $\beta$ compared with acute wounds. ${ }^{9}$ It has been suggested that growth factors may become trapped by ECM molecules ${ }^{10}$ or may be degraded by proteases to an excessive degree, ${ }^{11}$ resulting in nonhealing. An imbalance in chronic wounds between proteinases and their inhibitors with excessive proteinase activity, probably from over expression of matrix metalloproteins, results in degradation of the ECM. Many new treatment strategies are directed at modifying this imbalance to favour the healing process. These include topical application of proteinase inhibitors, inducing the expression of endogenous inhibitors or by combining proteinase inhibitors with growth factors. ${ }^{11}$ 
Senescent cells-Dermal fibroblasts have an age related decrease in proliferation potential, called senescence. ${ }^{12}$ Fibroblasts in chronic wounds have impaired responsiveness to growth hormone, which may be due to an increased number of senescent cells. ${ }^{13,14}$

\section{Cost implication of chronic wounds}

The management of chronic wounds places an enormous drain on healthcare resources. Cost to the NHS, UK in the 1990 's was estimated to be approximately $£ 1$ bn a year. ${ }^{15}$ In the United Kingdom, around 24000 patients with diabetic foot ulceration are admitted for treatment annually, costing the NHS $£ 17 \mathrm{~m} .{ }^{16}$ Foot ulceration is the commonest complication of diabetes that requires hospitalisation and in the United States, management of this problem is estimated to cost $\$ 150 \mathrm{~m}$ a year. ${ }^{17}$ Venous leg ulceration costs an estimated $£ 400 \mathrm{~m}$ annually in the United Kingdom, most of this cost being for dressings and community nurse visits. ${ }^{18}$

\section{Wound types}

\section{Venous ulcers}

Venous ulcers are the result of ambulatory venous hypertension with chronic venous insufficiency. Venous ulcerations generally develop on skin that typically exhibits the stigmata of chronic venous insufficiency such as hyperpigmentation, varicose eczema, lipodermatosclerosis or atrophie blanche. The skin around the ulcer therefore exhibits typical trophic changes of venous hypertension. Problems are caused by refractory ulcers that do not show signs of healing after 3 months or have not healed after 12 months of adequate treatment. ${ }^{19}$ Past trauma, immobility, deep vein thrombosis (DVT) are contributors to venous insufficiency. ${ }^{20}$ Factors such as weight gain and a sedentary occupation which contributes to

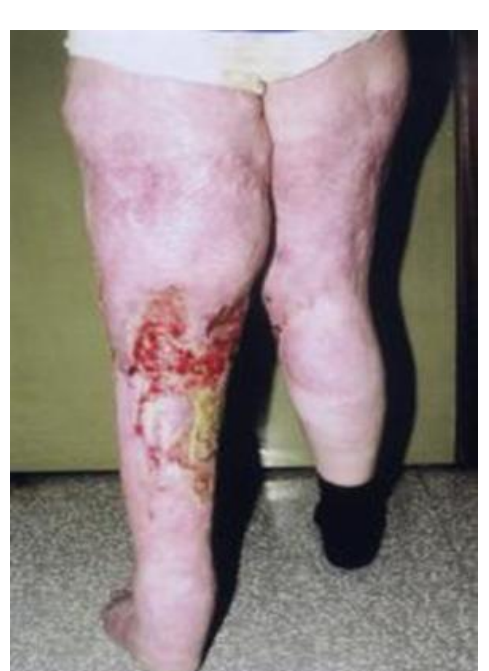

Figure 1 Lymphovenous leg ulceration with limb distortion. ${ }^{22}$ increased intra-abdominal pressure and negatively affects venous return to the heart should be checked. ${ }^{21}$ Along with varicose veins, brownish discolouration, oedema, lower leg dryness, signs of increased skin folds in the lower part of the limb and Stemmer's sign (a thickened skin fold at the base of the second toe) should be checked to exclude preexisting lymphatic insufficiency. $^{22}$

\section{Management of venous ulcers}

Following a proper clinical evaluation, identification of several clinical problems needs to be addressed to promote healing. These include oedema, irritant dermatitis, pain and tenderness, excessive exudate, purulent discharge, malodor, fungating growth, evidence of lymphedema, reduced mobility, disfiguration of the limb and discharging sinuses. Oedema is often a result of venous insufficiency, the site and nature of which may be determined using a duplex scan. Evidence of irritant dermatitis may suggest allergy to dressings or their constituents or some other reason where a dermatologist opinion would be helpful. The presence of pain, excessive exudate, seropurulent or purulent discharge and malodor may suggest infection and warrant microbiological investigations, including bacterial and fungal cultures. A fungating growth on a non-healing chronic ulcer may be evidence of carcinoma or actual fungal involvement and indicates the need for tissue biopsy from the site. Lymphedema suggests the extent of involvement of lymphatics. Reduced mobility of the limb and disfiguration and discharging 
sinuses may suggest the likelihood of chronic osteomyelitis or mycetoma. Radiological assessment, histology and fungal studies on biopsies is indicated to confirm the diagnosis. However, infection, malignancy and chronic osteomyelitis are uncommon in patients with venous ulceration. $^{22}$

Treatment regimen following specific measures mentioned above

Management of venous ulcers requires a multipronged approach. Control of the exudate requires hydrofiber dressings. When moisture is required in addition to absorption of excessive exudate, foam dressings are preferred. Antiseptic containing dressings are used when control of microbial flora is required and use of compression bandaging (using a multilayer short-stretch bandaging system) is helpful to prevent dependent oedema and leaking. Patients should be encouraged to be involved in appropriate cleansing and skin hydration using paraffin-based emollients. They should also be reminded of the risk of secondary infection and provided with information on hygienic measures that could help prevent infection. Specifically patients should be educated how to monitor for signs of infection at the ulcer site and for fungal infection between the toes. Physiotherapy, particularly when compression bandages are worn and provision of protective shoes too are an integral part of chronic wound management. Instruction on weight management for those who are obese would also be very helpful. In certain instances, specific exercises may be needed to improve normal functions of limbs and gait. ${ }^{22}$

\section{Maintenance management of venous ulcers}

The progress should be monitored at frequent intervals at the start and as required thereafter until successful recovery. Ulceration resulting from lymphovenous disease may require longterm management to prevent recurrence. The importance of early patient involvement in the management of chronic non-healing ulcers should be emphasized. ${ }^{22}$

\section{Diabetic ulcers,}

Although the aetiology of lower limb ulceration in patients with diabetes mellitus differs according to the population studied, ulcerated diabetic feet are a complex problem in all parts of the world. Ischaemia, neuropathy and infection are the three pathological components that lead to diabetic foot complications, and they frequently occur together as a triad. Neuropathy and ischaemia are the initiating factors, most often together as neuroischaemia and infection is mostly a consequence of neuroischaemia. The role of peripheral vascular disease in the diabetic foot ulcer has long been underestimated as typical ischaemic symptoms are less frequent in diabetics than in non-diabetics. In addition, the healing of a neuroischaemic ulcer is hampered by microvascular dysfunction. Therefore, the threshold for vascularising neuroischaemic ulcers should be lower than that for purely ischaemic ulcers. Previous guidelines have largely ignored these specific demands related to ulcerated neuroischaemic diabetic feet. Any diabetic foot ulcer should always be considered to have vascular impairment unless proven otherwise. ${ }^{24}$

Poor glycaemic control, previous ulcer/ amputation, neuropathy (abnormal sensorimotor perception of vibration), trauma (poorly fitting shoes, pressure, burns), altered biomechanics (limited joint mobility, bony prominences, foot deformity/osteoarthritis, callus), peripheral arterial occlusive disease, socioeconomic status resulting in poor access to medical facilities, lack of compliance/neglect and no or inadequate education are commonly encountered risk factors among patients who present with chronic diabetic ulcers. ${ }^{19}$

A population-based study in the north of England showed that $42 \%$ of Type 2 diabetic patients had clinical evidence of neuropathy. Trials have shown that the incidence of neuropathy in 
Type 1 diabetes can be reduced by over 50\% with intensive therapy and optimal glycaemic control. Hyperglycaemia is believed to be a major aetiological factor in the development of neuropathy in Type 2 diabetics. Neuropathy cannot be diagnosed through history alone. Careful examination of the feet for evidence of sensory loss and an assessment of the circulation must form part of the annual review of each patient. Peripheral somatic and autonomic neuropathy, together with peripheral vascular disease is a major contributing factor to the development of foot ulcers and therefore also needs evaluation. In addition, abnormalities of foot shape (e.g. claw toes, prominent metatarsal heads and shapes of arches of the foot) and the presence of plantar callus are signs of foot-ulcer risk. ${ }^{25}$

\section{Classification of diabetic foot ulcers}

There are many diabetic ulcer classifications. Many of those consider evidence of the extent of tissue damage such as the presence of callus or scar, superficial wound, wound penetrating to tendon or bone or necrosis of the foot. The presence of infection or ischaemia at all levels is also considered by some authors which is more complicated. ${ }^{19}$ As the ability to fight infection is reduced in hyperglycaemia, the microbiological assessment of diabetic ulcers is essential. The surface of wounds are invariably colonized or contaminated by large number of microbes. It is therefore necessary to obtain a deep swab or wound biopsy to determine the microbial load of a diabetic ulcer. Gram negative organisms, anaerobes and fungi are often associated with these ulcers.

\section{Assessment of diabetic foot ulcers}

Following a general evaluation of the patient, a complete vascular and neurologic assessment of the foot is required before further action. Further assessment of limb abnormalities and the level of ankle mobility are other supportive evidence required for proper management. Assessment of the level of ischaemia and the neuropathy deficit score is also important in the management of diabetic ulcers. The diagnostic criteria for diabetic neuropathy that are of particular relevance for diabetic foot syndrome is analogous to the Neuropathy Deficit Score (NDS) which includes examination of the Achilles tendon reflex and vibration, pain, temperature and touch perception. Examination of the Achilles tendon reflex with a reflex hammer is used to determine the depth sensitivity. Vibration perception is examined using biothesiometry which has a high predictive value for ulcer formation. The Rydel-Seiffer tuning fork is a simple and practical alternative to biothesiometry. It also makes sense to differentiate the neuropathic and arterial risk factors. ${ }^{26}$ Microbiological assessment involves the odour, colour, discharge in the wound bed and evidence of inflammation of the wound and around the wound.

\section{Management of chronic diabetic foot ulcers}

Early referral, non-invasive vascular testing, microbiological cultures, imaging and early intervention are crucial to improve diabetic foot ulcer healing and prevent amputation. Timing is essential, as the window of opportunity to heal the ulcer and save the leg is easily missed. There is an urgent need for a paradigm shift in diabetic foot care; that is, a new approach and classification of diabetics with vascular impairment in regard to clinical practice and research. A multidisciplinary approach needs to be implemented systematically. New strategies must be developed and implemented for the diabetic foot in patients with vascular impairment to improve healing, speed up the healing rate and avoid amputation, irrespective of the intervention technology chosen. Focused studies on the value of predictive tests, new treatment modalities as well as selective and targeted strategies are needed. ${ }^{24}$ As specific data on ulcerated neuroischaemic diabetic feet are scarce, recommendations are often of low grade. 
Effective patient education can reduce the incidence of foot ulceration and amputation by over $50 \%$. Therefore, all patients with a high risk of foot ulcers should be well informed on foot care and, if indicated, referred for regular podiatry. The team approach to diabetic foot problems is an effective method of providing treatment for active ulcers. This should be followed by appropriate education, the provision of follow up and if indicated, suitable footwear and hosiery. ${ }^{25}$ Almost all of these wounds are either infected or colonized or both, complicating the management of the patient. The primary team involve the surgeons (general, vascular, orthopaedic), physician (diabetologist) and the infectious disease consultant or clinical microbiologist. Dermatologists, pathologists, haematologists and oncologists may need to be consulted for specific problems as required. Health education plays a big role at all times. Motivation of the patient who is carrying an age old ulcer is often a difficult task. The level of education of the patient and various beliefs and attitudes may play a bigger role in low income countries in hindering patient involvement in the care of their own ulcer.

There are various new and well-designed dressings, growth promoters, antiseptics and antibiotics. ${ }^{27}$ The question in inadequately resourced health care systems is whether these can be provided free of charge or at minimal cost. ${ }^{25}$

\section{Pressure ulcers, assessment and management}

A pressure ulcer is a site of localized damage to the skin and/or the underlying tissue, usually over a bony prominence as a result of pressure, shearing forces, and/or friction. Pressure ulcers are divided into four stages based on the definitions of the European Pressure Ulcer Advisory Panel 1998.

\section{$\underline{\text { Stage } 1}$}

Non-blanchable erythema of the intact skin. Skin discoloration, warmth, oedema or induration can also be indicators of stage 1, particularly in dark-skinned individuals.

Stage 2

Partial-thickness loss of skin, with damage to the epidermis and/or the dermis. The ulcer is superficial and presents clinically as an abrasion or blister.

\section{$\underline{\text { Stage } 3}$}

Full-thickness loss of skin, including damage to or necrosis of the subcutaneous tissue that may extend to, but not through the fascia.

Stage 4

Extensive tissue necrosis possibly including muscle, bone or supporting structures

The most important endogenous risk factor for the development of a leg ulcer is peripheral sensorimotor neuropathy such as that which occurs in diabetes mellitus. The pressure relief by repositioning protects against a pressure ulcer. Mattresses with higher specifications have a greater protective effect than standard foam mattresses. There is no solid evidence verified by appropriate studies for diagnostic and therapeutic approaches but there are many expert opinions and comments. Changing positions, local massages or stimulations, including the use of electrical stimulation has demonstrably enhanced wound healing in pressure ulcers. ${ }^{28}$

Prevention of pressure damage to the skin and the underlying tissue is an essential part of treatment in at-risk patients. Adequate risk assessment and subsequent risk reduction is crucial.

Surgical strategies should be considered for pressure ulcers from stage II onwards that do not heal using conservative measures. If no tendency to heal becomes apparent, surgical debridement with the aid of the previously mentioned conservative measures and plastic 
surgery reconstruction is indicated where appropriate. Surgical measures must be planned and undertaken in the context of the general condition of the patient. Geriatric patients in particular often suffer from multiple comorbidities. For local treatment, absolute pressure relief is critical for wound healing. In addition, guidelines prepared for the management of chronic venous ulcers provide assistance in planning treatment strategies for longstanding and non-healing pressure ulcers. Appropriate pressure relief has priority for neuropathic, diabetic or pressure ulcers. $^{25,26}$

\section{Microbiology of chronic wounds}

Venous ulcers, diabetic ulcers and pressure ulcers are the most commonly encountered ulcers in day to day practice. In addition, chronic ulcers could result following surgery of compound fractures and failed skin grafts. These may be complicated by diabetes, venous insufficiency or some other factors. All these wounds are of long lasting nature and invariably lead to recurrent cellulitis or infections which further lengthen the time for healing. The prevention and appropriate treatment of infections therefore has an important role in chronic wound management. However, frequent antiseptic use, secondary irritant dermatitis or any other local or systemic allergies may also contribute towards the chronicity of the wounds. Most wounds of greater than 2 years duration are usually associated with almost every risk factor mentioned above singly or repeatedly during the course of the patient's period of illness as seen even in Sri Lankan clinics.

All chronic wounds are contaminated by bacteria, even though wound healing could occur in the presence of bacteria. These contaminants could either be derived from personal indigenous microflora and/or from the environment. It is interesting to note that most contaminating organisms in chronic wounds are not able to multiply in a wound. Most of the colonizing organisms are from adjacent normal skin. They are Staphylococcus epidermidis, and other coagulase negative staphyococci, Corynebacterium spp., Brevibacterium spp., Proprionibacterium acnes, and Pityrosporum spp. It is not the presence of organisms, but their interaction with the patient and the wound milieu that determines their influence on wound healing. ${ }^{30}$

Wound infection is caused primarily by Staphylococcus aureus, $\beta$-hemolytic streptococci (Streptococcus pyogenes, S. agalactiae) and anaerobes. Enterobacteria (Escherichia coli, Proteus spp., Klebsiella spp.) and Gram negative non fermenters (Pseudomonas spp., Acinetobacter spp. Stenotrophomonas spp.) often colonize wounds and although not a direct cause of wound infection, may sometimes contribute to it. ${ }^{30}$

The microbial flora in wounds appears to change over time. Normal skin flora predominates in early acute wounds. $S$. aureus and $\beta$ haemolytic streptococci soon follow. Group B $\beta$ haemolytic streptococcus and $S$. aureus are commonly found in diabetic foot ulcers. After about 4 weeks, facultative anaerobic gram negative bacilli (most commonly Proteus spp., E. coli and Klebsiella spp.) will colonize the wound. As the wound deteriorates, deeper structures are affected and anaerobes become more common. Often such infections are polymicrobial. Long-term chronic wounds often contain more anaerobes than aerobes. Aerobic gram negative rods (Pseudomonas spp., Acinetobacter spp., Stenotrophomonas spp.) also infect wounds late in the course of chronic wounds. These are usually acquired from exogenous sources such as bath and foot water. Organisms like Pseudomonas spp. are usually not very invasive unless the patient is highly compromised, such as with severe neutropaenia, where it could cause ecthyma gangrenosum. However, these organisms are associated with 
marked wound deterioration due to endotoxin, enzymes, and exotoxin production. As the wounds go deeper and become more complex, the underlying muscles and bone may get involved in the infective process, causing osteomyelitis. In addition to $S$. aureus, coliforms and anaerobes are associated with osteomyelitis in these patients. Enterococcus and Candida spp. are often isolated from wounds. Treating a patient for these organisms is only indicated if there are no other pathogens present and the organisms are present in high concentrations of $>10^{6} \mathrm{CFU}$ 's per gram of tissue.

Many factors affect the progress of microorganisms in a wound from colonization to infection including the number of organisms, the virulence factors they produce and the resistance of the host to infection.

The number of organisms required to initiate infection differs with the type of organism. Some organisms such as Candida spp. and enterococci need to be present in high concentrations. Dual infections as in the case of infection by $S$. agalactiae and S. aureus may result in more host damage due to synergy. The primary pathogens which are present on wound surfaces such as $\beta$ haemolytic streptococci, mycobacteria spp., Bacillus anthracis, Yersinia pestis, Corynebacterium diphtheriae, Erysipelothrix rhusiopathiae, Leptospira spp., Treponema spp., Brucella spp., Clostridium spp., Varicella Zoster Virus (VZV), Herpes Simplex Virus (HSV), dimorphic fungi and Leishmania spp. require treatment irrespective of the count. ${ }^{31}$

Infection by $S$. pyogenes, owing to its multitude of virulence factors could result in necrotizing fasciitis or streptococcal toxic shock syndrome, which though not very common, are associated with high morbidity and mortality. Cellulitis and erysipelas are a more common manifestation of infection of a chronic wound with S. pyogenes. E. rhusiopathiae, associated with hog farmers and people who comes into contact with water frequently, such as fishermen, can also infect chronic wounds. Mycobacterium marinum and M. ulcerans can infect chronic wounds and should be thought of when patients with chronic ulcers are associated with aquaria, pools or go fishing. ${ }^{29}$

Virulence factors produced by bacteria contribute to host damage and increased mortality, some examples being hyaluronidase ( $S$. pyogenes), proteases ( $S$. aureus, $P$. aeruginosa), toxins (S. pyogenes, S. aureus) and endotoxin (gram negative bacteria).

Local factors that increase the possibility of wound infection are large wound area, increased wound depth, degree of chronicity, anatomic location (distal extremity, perineum), foreign body, necrotic tissue, mechanism of injury (bites, perforated viscera) and reduced perfusion.

Systemic factors that increase the possibility of wound infection are vascular disease, oedema, malnutrition, diabetes, chemotherapy, alcoholism, prior surgery or radiation, corticosteroids and inherited neutrophil defects

Figure 2 shows the different organisms involved at different structures of the skin and soft tissue. ${ }^{30}$ One feature common to all infected chronic wounds is the failure of the wound to heal and progressive deterioration of the wound overtime. Although infection of the wound is not the sole reasons for poor wound healing, it makes a significant contribution to poor healing.

Typical features of wound infection include an increase in exudate, swelling, erythema, pain and local temperature, peri-wound cellulitis, ascending infection and change in the appearance 
of the granulation tissue which is discoloured, prone to bleed and highly friable. Those microorganisms that have been mentioned earlier which would cause acute infections can be controlled by appropriate antibiotic therapy. However organisms such as Pseudomonas spp, known to have biofilms,. seem to be identified as most troublesome to control as encountered in cystic

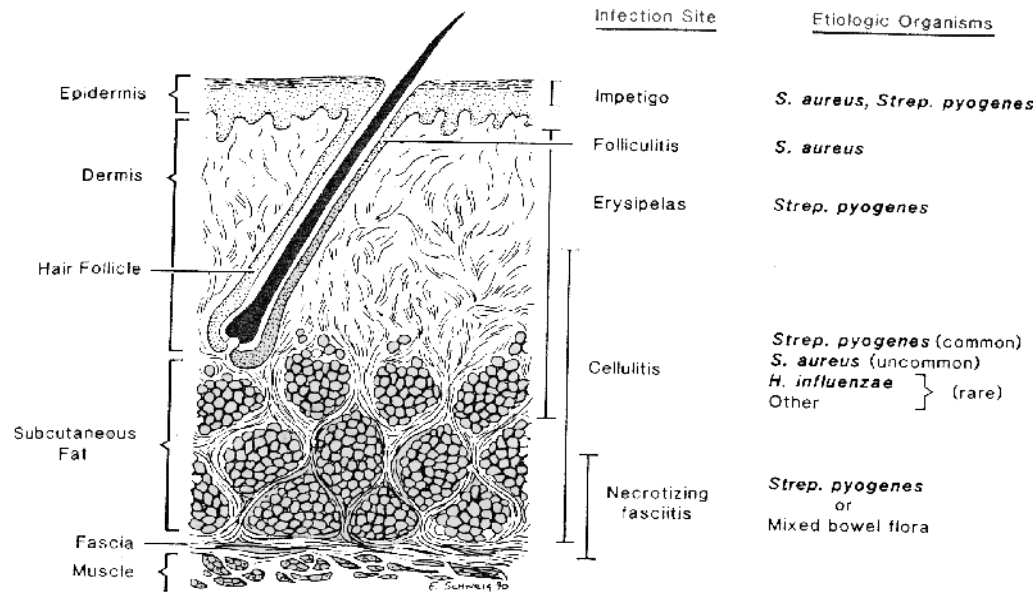

Figure 2 :Skin structure and organisms commonly involved at different levels ${ }^{30}$ fibrosis. ${ }^{31}$

It has also been shown that inhibition of epithelialization and migration of keratinocytes occurs through nuclear localization of $\beta$-catenin and elevated c-mycexpression at the non healing edge of chronic ulcers. This altered molecular pathway may explain delayed wound healing. ${ }^{32}$

Bacterial culture helps to determine the aetiological agents of an infection when the sample is taken properly from the area most representing infection. The gold standard for sample collection is to do a tissue biopsy or needle aspirate from the leading edge of the wound after debridement. The specific anatomic site of the biopsy and the nature of the wound (surface or deep) should be indicated in the request form. Gram stain of a smear of the tissue should be requested. Surface wounds are NOT cultured for anaerobes whereas anaerobic cultures of deep wounds are useful. If a tissue biopsy is not possible, a swab taken from the base of the lesion after vigorous cleansing of the wound with sterile saline is advised. Swabs taken from surface wounds are transported to the laboratory in a sterile container. It is best to transport swabs from deep wounds in a sterile anaerobic container if anaerobic culture facilities are available in the receiving laboratory. Quantitative culture of tissue should be carried out if possible as greater likelihood of sepsis is associated with $>10^{5} \mathrm{CFU} / \mathrm{gm}$ of tissue. ${ }^{30}$

\section{Advances in microbe control in wounds}

Bacteriophages can be an effective topical therapy against $S$. aureus biofilm-infected wounds in the setting of a deficient (mutant) or disrupted (debridement) biofilm structure. Combination treatment, aimed at disturbing the extracellular biofilm matrix and allowing for increased penetration of species-specific bacteriophages, represents a new and potentially effective approach to chronic wound care. These results establish principles for biofilm therapy that may be applied to several different clinical and surgical problems. ${ }^{33}$

The use of topical antiseptics lacks adequate proof of efficacy and residual concerns of their potential toxicity to healing wounds which may impair wound healing. The risk for local toxicity depends on the ingredients contained in the formulations, the concentration of active ingredient and the duration of contact of the active ingredient. Newer formulations and methods of applying topical antiseptics appear to reduce the risk of interference with the healing process. Antiseptics should not be used in solutions because they are more likely to 
cause cell damage and have not demonstrated benefit over saline irrigation. Newer topical creams, ointments, gels, and dressings appear to provide adequate, sustained, and apparently nontoxic levels of antiseptics. Unfortunately, there is little information on systemic absorption of the agents and evidence of clinical efficacy is meagre. Thus, clinicians should currently use these products very selectively and only for a short duration. Investigators and the industry are seeking other ways to deal with chronic wound infections, including various innovative non antimicrobial approaches such as Bee honey and Aloe vera. ${ }^{34-39}$

Topical antiseptics, such as sustained release silver and honey provide some evidence of their value in managing biofilms. Empirically, several researchers have noted that iodine preparations, particularly cadexomer iodine, also possess the capability to manage biofilm infection. ${ }^{40-43}$ However, when used concomitantly with frequent debridement and other topical agents that impair biofilm defences, antibiotics can be more successful. Clinical medicine has found that for biofilm diseases such as osteomyelitis and endocarditis, higher doses of antibiotics for longer periods of time are more effective. In a chronic wound, use of antibiotics as a single agent struggles to suppress biofilm, but when used in conjunction with the other strategies indicated above, does show significant impact on the healing process. Because wound biofilms are resistant to antibiotics and host defences, clinicians struggle to successfully manage many chronic wounds. Aggressively targeted wound biofilm suppression and reduction in the bio burden for a period of time is needed until the host immune response prevails and resolves the chronic wound. ${ }^{44-47}$

In the light of the extent and importance of the problem of chronic wound infection, it is mandatory to do further research on non-antimicrobial as well as antimicrobial products. In addition, frequent debridement sets the stage for therapeutic agents to be more effective. Debridement provides a cornerstone in the management of chronic wounds and evidence demonstrates that frequent debridement improves wound healing. ${ }^{48,49}$

As a clinical microbiologist, my interest in the management of chronic wounds was stimulated with the successful management of a patient with a chronic wound associated with actinomycosis which extended to the bone and 2 patients with chronic osteomyelitis. Microbiological investigations were crucial in designing their treatment plan. This resulted in initiation of a chronic wound management (CWM) clinic in the hospitals where I have served since then. Isolation and identification of both bacteria and fungi from samples taken from chronic wounds resulted in consultation with clinical colleagues as well as inputs into the management of patients with such wounds. Consultant colleagues as well as the administrative Head of the respective institutions actively encouraged and supported the setting up of CWM clinics in three hospitals. The clinic in one of the hospitals serves only inpatients whereas in both other centres, patients are referred by the outpatient department of the hospital.

The clinics are run according to an agreed pattern. General and local assessments are carried out on patients at their first visit. A swab for direct smear and basic bacterial culture is taken to assess the nature of colonizers of the wound. If the clinical assessment is suggestive of an infection, further cultures are taken as appropriate. Patients with severe infections are admitted for inpatient treatment. The rest are managed with appropriate oral antibiotics. Antiseptic choice, type and frequency of dressing are based on primary culture results.

Patients are monitored weekly. Assessment at each visit includes examination of the wound bed, peri-wound, presence or absence of discharges and irritant dermatitis. 
General investigations including full blood count, fasting blood sugar, liver function tests, renal function tests such as blood urea and serum creatinine and $\mathrm{C}$ reactive protein are done on all patients. Surgical biopsies are also performed when indicated. Where required, patients are referred to other specialities for assessment and inputs on management.

Initially, patients were referred to resourced hospitals for wound debridement and surgical (vascular, general, orthopaedic) care when needed. Venous duplex studies also needed to be done at such hospitals prior to referral to surgeons. With the appointment of a full time general surgeon to one of the hospitals, a combined approach locally has improved the management of patients considerably.

Patients seen at the clinics have chronic wounds of varying duration, ranging from 4 months to 20years. Venous and diabetic ulcers are the commonest with venous ulcers being the more common. Pressure ulcers, chronic osteomyelitis, chronic recurrent fungal and bacterial mixed nail infections, infected eczemas, elephantiasis (filarial and non filarial), mycetomas and ulcers in patients with haematological disorders are some of the many underlying causes of chronic wounds seen at these clinics (unpublished data). Laboratory based studies on the microbiological properties of Sri Lankan Bee Honey ${ }^{50}$ and other studies are in progress.

In Sri Lanka, there is insufficient information on the extent of the problem of chronic wounds as well as the health budget required for managing such wounds. However, using data generated from these 3 clinics, the average cost per day for a routine wound dressing is about Rs $250 /=$. Patients either come daily or every other day for wound care for periods extending from 2 to over 20 years. The magnitude of health costs incurred can therefore be estimated for an individual hospital. The direct costs to the institution does not reflect the frustration, economic loss, and impaired quality of life experienced by patients with chronic ulcers.

Management of chronic wounds involve the management of multiple local and systemic underlying conditions. Proper assessment, identifying all the problems, multidisciplinary involvement for management and regular monitoring is required for healing as well as prevention of recurrences. The very positive responses of colleagues and hospital management staff to the wound management clinics at 3 hospitals have improved patient management as well as outcomes of chronic wounds. This provides a model for discussion and possible action for those who are responsible for the management of these patients in Sri Lanka

\section{References}

1. BowlerPG, Duerden BI, ArmstrongDG.:Wound microbiology and associated approaches to wound management.Clin. Microbiol. Rev. 2001;14(2):244-69 doi: 10.1128/CMR.14.2.244-269.2001.

2. Yamaguchi Y, Yoshikawa K.Cutaneous wound healing: an update.The Journal of Dermatology 2001;28(10): 521-34 No doi

3. Yamada KM and Clark RAF. Provisional matrix. In: The Molecular and Cellular Biology of Wound Repair. Clark, RAF ed. Plenum Press, New York, NY, 1996, pp 51-93.

4. Schultz GS, SibbaldRG, Falanga V, et al.Wound bed preparation: A systematic approach to wound management.Wound RepairRegen 2003;11:1-28

http://dx.doi.org/10.1046/j.1524-475X.11.s2.1.x 
5. Cooper DM, Yu EZ, Hennessey P, Ko F, RobsonMC. Determination of endogenous cytokines in chronic wounds.Ann Surg 1994;219:688-92. http://dx.doi.org/10.1097/00000658-199406000-00012

6. Clark RAF.Biology of dermal wound repair.Dermatologic Clinics1993;11:647-66. No doi

7. Mauch C, Krieg T, Bauer EA.Role of the extracellular matrix in the degradation of connective tissue.Archives of Dermatological Research1994; 287:107-14. http://dx.doi.org/10.1007/BF00370728

8. Harding KG, Morris HL, Patel GK. Healing chronic wounds.BMJ 2002;324:160-63 http://dx.doi.org/10.1136/bmj.324.7330.160

9. Higley HR, Ksander GA, Gerhardt CO, FalangaV. Extravasation of macromolecules and possible trapping of transforming growth factor-beta in venous ulceration. $\mathrm{Br} J$ Dermatol1995;132:79-85.http://dx.doi.org/10.1111/j.1365-2133.1995.tb08629.x

10. Bucalo B, Eaglstein WH, Falanga V.Inhibition of cell proliferation by chronic wound fluid.Wound Repair Regen1993;1:181-86. http://dx.doi.org/10.1046/j.1524475X.1993.10308.x

11. Barrick B, Campbell EJ, Owen CA. Leukocyte proteinases in wound healing: roles in physiologic and pathological processes.Wound Repair Regen1999;7:410-22. http://dx.doi.org/10.1046/j.1524-475X.1999.00410.x

12. Agren MS, Steenfos HH, Dabelsteen S, HansenJB, DabelsteenE.Proliferation and mitogenic response to PDGF-BB of fibroblasts isolated from chronic venous leg ulcers is ulcer-age dependant.J Invest Dermatol1999;112(4): 463-69. http://dx.doi.org/10.1046/j.1523-1747.1999.00549.x

13. Hasan A, Murata H, Falabella A, Ochoa S, ZhouL, Badiavas E, et al. Dermal fibroblasts from venous ulcers are unresponsive to the action of transforming growth factor- $\beta$. Journal of Dermatological Science 1997;16(1):59-66. http://dx.doi.org/10.1016/S0923-1811(97)00622-1

14. Stanley AC, Park HY, Phillips TJ, RussakovskyV,Menzoian JO. Reduced growth of dermal fibroblasts from chronic venous ulcers can be eliminated with growth factors. $J$ VascSurg1997;26(6):994-99. http://dx.doi.org/10.1016/S0741-5214(97)70012-0

15. Harding KG: The future of wound healing. In: Leaper DJ, Harding KJ, editors. Wounds: biology and management. Oxford: Oxford University Press; 1998. p. 191. ISBN 019262332X, 9780192623324

16. Currie CJ, Morgan CL, Peters JR.The epidemiology and cost of inpatient care for peripheral vascular disease, infection, neuropathy and ulceration in diabetes. Diabetes Care 1998;21:42-8.doi:10.2337/diacare.21.1.42

17. Reiber GE. Diabetic foot care: financial implications and practical guidelines.Diabetes Care1992;15(suppl 1):29-31doi:10.2337/diacare.15.1.S29

18. Ruckley CV. Socio-economic impact of chronic venous insufficiency and leg ulcers.Angiology 1997;48:67-9. No doi

19. Birgit Kahle P.D. Hans-Joachim Hermanns, Georg Gallenkemper. Evidence based treatment of chronic leg ulcers.DtschArztebl Int. 2011;108(14):231-37doi: 10.3238/arztebl.2011.0231

20. Callam MJ, Harper DR, Dale JJ, Ruckley CV. Chronic ulcer of the leg: clinical history. BMJ1987;294(6584):1389-91.http://dx.doi.org/10.1136/bmj.294.6584.1389

21. Adhikari A, Criqui MH, Wooll V et al. The epidemiology of chronic venous disease. Phlebology 2000;15(1):2-18.http://dx.doi.org/10.1007/s005230070031

22. Browse N, Burnand KG, Mortimer PS. Diseases of the Lymphatics. London: Hodder Arnold, 2003ISBN-13: 978-0340762035 
23. Debra Doherty. World Wide Wounds, Revision 1, Nov 2005 accessed at http://www.worldwidewounds.com/2005/november/Doherty/Encourage-patient-InvolvementMgt-Lymphovenous.html

24. 25Lepäntalo M, Apelqvist J, Setacci C, Ricco JB, de Donato G, Becker F et al. Chapter V-Diabetic foot. Eur J VascEndovascSurg 2011;42(suppl2):S60-4 doi: 10.1016/S1078-5884(11)60012-9

25. 26Boulton AJ. Lowering the risk of neuropathy,foot ulcers and amputation. Diabet Med 1998;15(S4): S57-9http://dx.doi.org/10.1002/(SICI)1096-9136(1998120)

26. Young MJ,BreddyJL,Veves A, Boulton AJM. The prediction of neuropathic foot ulceration using vibration perception thresh holds. Diabetes Care 1994;17(6):55761http://dx.doi.org/10.2337/diacare.17.6.557

27. 24Wanless D. Securing Our Future Health: Taking a Long-Term View. Final Report. London: HM Treasury, 2002.

28. Macdonald JM, Gayer MJ.Ed Wound and Lymphoedema management. WHO/HTM/NTD/GBUI/ 2010.1ISBN 9789241599139

29. Manesh A, Flemming K, Cullum NA, Ravagi H. Electromagnetic therapy for treating pressure ulcer. Cochrane Database of Systematic Reviews2006:(Issue 2)

Art.No:Cd002930.doi 10.1002/14651858.CD002930.Pub 3

30. Dow G, Browne A, Sibbald RG. Infection in chronic wounds: controversies in diagnosis andtreatment. Ostomy Wound Manage 1999; 45(8):23-40 No doi

31. Bjarnsholt T,Kerketerp-Moller K,Jenson PO et al.Why chronic wounds will not heal: a novel hypothesis. Wound repair Regen 2008;16(1): 210http://dx.doi.org/10.1111/j.1524-475X.2007.00283.x

32. Stojadinovik O,Brem H,Vouthounis Cet al.Molecular Pathogenesis of chronic wounds- The role of $\beta$ Catenin and C-myc in the inhibition of epithelialization and wound healing. Am J Pathol. 2005;167(1):59-69 No doi

33. Seth AK, Geringer MR, NquyenKTet al.Bacteriophage therapy for Staphylococcus aureus biofilm-infected wounds: a new approach to chronic wound care. PlastReconstrSurg 2013;131(2):225-34http://dx.doi.org/10.1097/PRS.0b013e31827e47cd

34. Cooper R. A review of the evidence for the use of topical antimicrobial agents in wound care. World Wide Wounds. 2004. February:1-11. Available at http://www.worldwidewounds.com/2004/february/Cooper/Topical-Antimicrobial-Agents.html. Accessed 7 October 2009.

35. O'Meara S, Al-Kurdi D, Ovington LG. Antibiotics and antiseptics for venous leg ulcers. Cochrane Database Syst Rev. 2008;(1):CD003557.doi: 10.1002/ 14651858

36. Bergin SM, Wraight P. Silver based wound dressings and topical agents for treating diabetic foot ulcers. 2006 Cochrane Database Syst Rev. 2006;(1):CD005082. doi:10.1002/14651858.CD005082.pub2

37. Jull AB, Walker N, Deshpande S. Honey as a topical treatment for wounds. Cochrane Database Syst Rev2008;(4):CD005083. doi: 10.1002/14651858.CD005083.pub3

38. Neely AN, Gardner J, Durkee P, et al. Are topical antimicrobials effective against bacteria that are highly resistant to systemic antibiotics? J Burn Care Res 2009;30(1):19-29http://dx.doi.org/10.1097/BCR.0b013e3181921eed

39. White RJ, Cutting K, Kingsley A. Topical antimicrobials in the control of wound bioburden. Ostomy Wound Manage 2006;52(8):26-58. No doi

40 Percival SL, Bowler PG, Woods EJ. Assessing the effect of an antimicrobial wound dressing on biofilms. Wound Repair and Regeneration 2008;16:52-7 http://dx.doi.org/10.1111/j.1524-475X.2007.00350.x

41 Chaw KC, Manimaran M, Tay FEH. Role of silver ions in destabilization of intermolecular adhesion forces measured by atomic force microscopy in 
Staphylococcus epidermidis biofilms. Antimicrobial Agents and Chemotherapy 2005; 49 (12): 4853-59. http://dx.doi.org/10.1128/AAC.49.12.4853-4859.2005

42 Okhiria $\mathrm{O}$, Henriques $\mathrm{A}$, Burton $\mathrm{N}$, et al. The potential of manuka honey for the disruption of biofilms produced by strains of Pseudomonas aeruginosa isolated from wounds. Poster presentation at 155th meeting of Society for General Microbiology meeting in Dublin 6-9th September 2004 No doi

43 Irish J, Carter D, Blair S. Honey prevents biofilm formation Staphylococcus aureus. Poster presentation - 8th Asian Apicultural Association Conference, Perth, Australia, 20-24th March, 2006.No doi

44 Stewart PS. Theoretical aspects of antibiotic diffusion into microbial biofilms. Antimicrobial Agents Chemotherapy 1996; 40: 2517-22.No doi

45 Fux CA, Costerton JW, Stewart PS, Stoodley P. Survival strategies of infectious biofilms. Trends in Microbiology 2005; 13:34-40. http://dx.doi.org/10.1016/j.tim.2004.11.010

46 Stewart PS, Roe F, Rayner J, et al. Effect of catalyse on hydrogen peroxide penetration into Pseudomonas aeruginosa biofilms. Applied and Environmental Microbiology 2000; 66(2):836-8.http://dx.doi.org/10.1128/AEM.66.2.836-838.2000

47 Leid JG, Willson CJ, Shirtliff ME, et al. The exopolysaccharide alginate protects Pseudomonas aeruginosa biofilm bacteria from IFN gamma-mediated macrophage killing. Journal of Immunology 2005;175:7512-18.No doi

48 Armstrong PA, Back MR, Bandyk DF, et al. Selective application of Sartorius muscle flaps and aggressive staged surgical debridement caninfluence long-term outcomes of complex prosthetic graft infections.Journal of Vascular Surgery 2007;46(1):71-8. http://dx.doi.org/10.1016/j.jvs.2007.02.058

49 Steed DL, Donohoe D, Webster MW, Lindsley L. Effect of extensivedebridement and treatment on the healing of diabetic foot ulcers. Diabetic Ulcer Study Group. Journal of the American College of Surgeons 1996;183(1):61-4 No doi

50 WadanambyJMRWW, Caldera RCK, Lankika I. An effort to understand the microbiological properties of Sri Lankan Bee Honey, Poster presentations, Annual Scientific Sessions of Sri Lanka Medical Association 2012\& Sri Lanka College of Microbiologists 2012 No doi 